\title{
Dynamic behavior model of the ballastless railroad track segment considering wave processes
}

\author{
Alexey Loktev ${ }^{1}$, Vadim Korolev ${ }^{1}$, Irina Shishkina ${ }^{1 *}$, Alexander Savin ${ }^{1}$, Liliya Illarionova ${ }^{1}$, \\ Olga Lokteva ${ }^{1}$, and Vladislav Kuskov ${ }^{1}$ \\ ${ }^{1}$ Russian University of Transport (MIIT), Chasovaya str. 22/2, Moscow, 125190, Russia
}

\begin{abstract}
The paper is devoted to the modification of the dynamic behavior model of a flat orthotropic element under shock and vibrational impacts. The impacts were caused by solid cylindrical body for the case of ballastless railway construction. The suggested model allows simulating the element deformation in the upper structure of the ballast-free design track. The propagation of elastic waves moving with finite speeds, orthotropic properties of the foundation slab, which allow optimally selecting rod reinforcement are taken into account. The obtained analytical and graphical results make it possible to assess the influence of properties of the reinforced concrete slab, the underframe speed, the presence of defects in wheel sets or rail bars, as well as the characteristics of the technical content of the track section.
\end{abstract}

\section{Introduction}

To date, the most common and generally accepted is the design of the railway track on a ballast base in the form of a rubble prism. This structure usually consists of rails, tie bars and rubble or sand base (Fig. 1a). Low construction costs, good maintainability and operational durability determine the use of the ballast track almost everywhere in Russia and abroad. Moreover, for several decades, such design was considered the only possible one. However, with increasing speeds, the emergence of high-speed transport with a range of speeds from $40-200 \mathrm{~km} / \mathrm{h}$, with a traffic increase and tightening requirements for quality and track safety, solutions for modernizing the existing track laying technology are being sought [1]. The maximum speed that rolling stock can gather often exceeds the capabilities of existing railways. This is one of the reasons why reconstructing and constructing new tracks are relevant and timely [2]. The study is devoted to developing a model of the dynamic behavior of the ballastless track (Fig. 1b) under the action of rolling stock. The model should take into account the anisotropic properties and wave processes occurring in the structure after shock and vibration loads $[3,4,5]$.

The track system on a ballastless foundation has mostly two following forms: with a solid concrete foundation or with a rail resting on a reinforced concrete slab. The type of

\footnotetext{
* Corresponding author: shishkinaira@inbox.ru
} 
track on a reinforced concrete continuous rail foundation does not require preliminary assembly of track elements $[6,7,8]$. When implementing this technology, the rail tops and shapes are installed in the working position. After the final alignment, concrete mixture is poured around the rail supports, which fixes the rails in the required position $[9,10,11]$. The option when rails are rested on a precast reinforced concrete slab does not require additional costs for a large amount of concrete work, and is also easier to repair, which is more cost-effective.

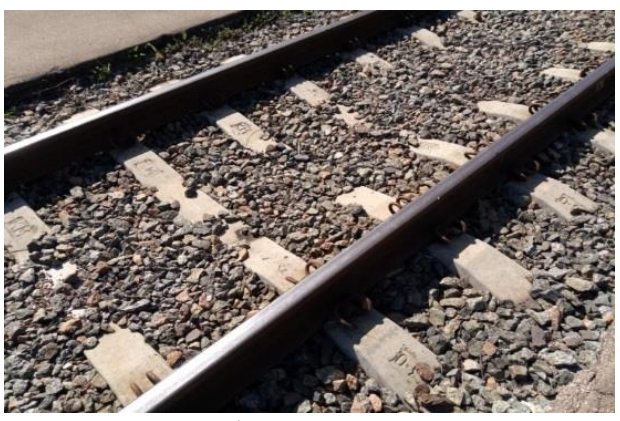

a)

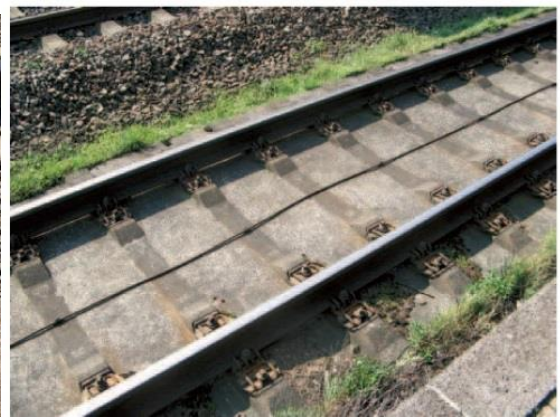

b)

Fig. 1. The upper structure of the railway: a) ballast track panel, b) ballastless structure

The advantages of ballastless track design are as following:

- lack of vegetation, therefore, the need to combat it is excluded;

- ease of use on overpasses, bridges and in tunnels;

- the structure has a high own weight, thereby ensuring good stability;

- no threat of ballast particles departing from the rolling stock when moving at high speeds that could harm rolling stock and infrastructure elements;

- the advantage of ballastless design regarding the stability of the jointless track to thermal emission;

- better durability of the track with minimal maintenance costs;

- increase of the operational speed of trains.

The amount of lump sum funds invested in the construction of a ballastless track is compensated by a decrease in maintenance costs and an increase in the operating life [12].

The movement of rail transport along the railway causes fluctuations in the upper structure of the track, as well as vertical movements of rail points $[13,14,15,16]$. After testing the integrity of the concrete slab and the elements of the rail fasteners, cracks were found in the upper concrete slab and local concrete spalls in contact with half ties (Fig. 2).

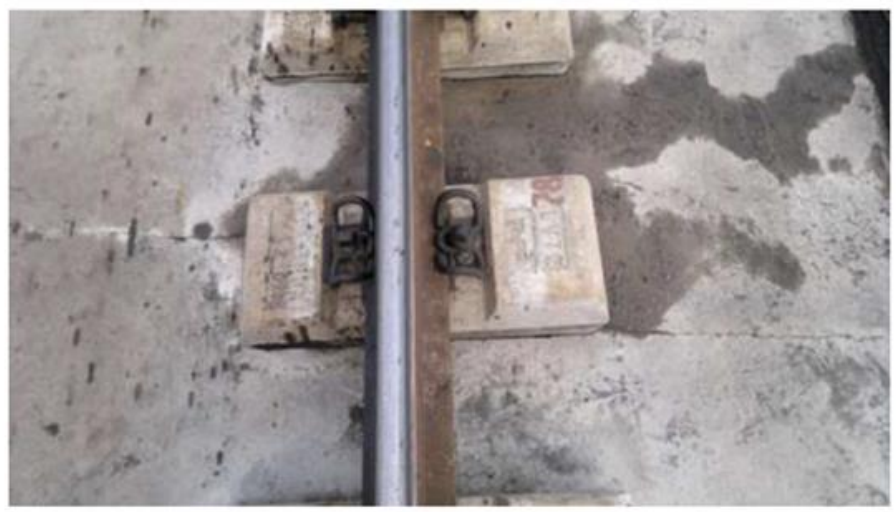

Fig 2. Cracks in the reinforced concrete foundation slab 


\section{Research technique}

The goal of the research is to check the local strength of the slab undergoing shock impact, as well as to study its influence on the dynamic deflection of the plate. The effect on contact force of the cylindrical anisotropy and on the initial velocity of interaction impact was also studied, the rigidity conditions of the plate was checked $[17,18,19]$. The problems of transverse axisymmetric impact of a solid body on an orthotropic plate with cylindrical anisotropy are solved [20, 21, 22]. The dynamic behavior of the plate is described by nonclassical equations of the Ufland-Mindlin type, which include five movements of the target points [23, 24]. The effect of the anisotropic, thermoelastic properties of the plate material is studied, as well as the effect of prestress on the propagation velocity of wave surfaces, dynamic deflection and contact force at the point of interaction and stress at arbitrary target points.

The deformation of the elements of the track upper structure in case of ballastless design occurs also due to the propagation of elastic waves moving with finite velocities [25, 26].

The interaction of a wheel pair and a rail at a given track point is characterized by the appearance of a contact region with $r_{0}$ radius. Starting from the boundary of this region, a quasi-longitudinal wave begins to propagate, at the front of which tensile-compression strains predominate, as well as a quasi-transverse wave, at the front of which shear strain is predominant. In Fig. 3, the following notation was adopted: $P(t)$ is the contact force between the wheel pair and the rail, $Q_{r}$ is the transverse force acting along the contour of the contact area, $\alpha$ is the buckling of the wheel and rail materials in the contact zone, $w$ is the deflection of the rail bar between two adjacent half ties, $w_{1}$ - movement of the lower rail edge, $w_{2}$ - movement of the lower edge of the half ties.

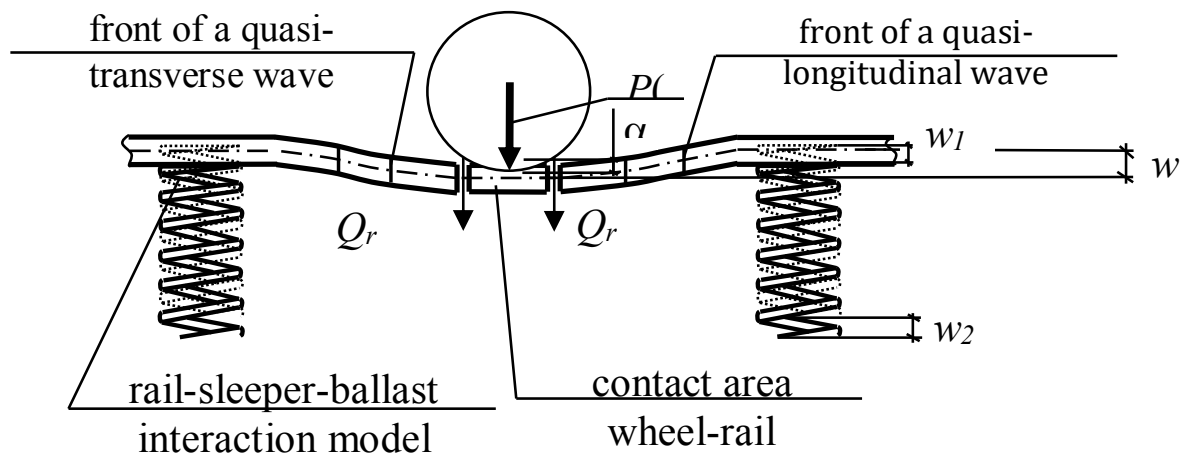

Fig. 3. Contact model of the wheel and the upper track structure

In order to determine the values responsible for the dynamic deformation of the upper track structural elements, it is necessary to compile a system of equations. Such system should characterize the behavior of the wheelset, the upper track structure and the contact area after the start of interacting with the rolling stock [27, 28].

Taking into account the equation describing the vertical movement of the wheel pair, the motion equation of the contact area at the boundaries shown in Fig. 3, it is clear that the equation system is required. The condition of the horizontal tangent to the middle surface of the plate at the boundary points of the contact area is also considered. Such an equations system should determine the interaction process between the wheel pair and the slab top structure of the track:

$$
\begin{gathered}
m(\ddot{\alpha}+\ddot{w})=-P(t), \\
\rho h \pi r_{0}^{2} \ddot{w}=2 \pi r_{0} Q_{r}+P(t),
\end{gathered}
$$




$$
\left|\frac{\partial W}{\partial r}\right|_{r=r_{0}}=0,
$$

In expression (1), the relation for the contact force $P(t)$ depends on the dynamic contact model of the wheel and rail (elastic, viscoelastic, nonlinear elastic, elastoplastic, etc.).

The dynamic behavior of an elastic orthotropic plate of the Ufland-Mindlin type is described by equations in the polar coordinate system. Such plate is most suitable for modeling a reinforced concrete slab of a ballastless track regarding the number of considered parameters. These equations take into account the rotation inertia of the cross sections, the strain of the transverse shear and the axisymmetry of the loading issue in the straight track section:

$$
\begin{gathered}
D_{r}\left(\frac{\partial^{2} \varphi}{\partial r^{2}}+\frac{1}{r} \frac{\partial \varphi}{\partial r}\right)-D_{\theta} \frac{\varphi}{r^{2}}+h K G_{r z}\left(\frac{\partial w}{\partial r}-\varphi\right)=-\rho \frac{h^{3}}{12} \frac{\partial^{2} \varphi}{\partial t^{2}} \\
K G_{r z}\left(\frac{\partial^{2} w}{\partial r^{2}}-\frac{\partial \varphi}{\partial r}\right)+K G_{r z} \frac{1}{r}\left(\frac{\partial w}{\partial r}-\varphi\right)=\rho \frac{\partial^{2} w}{\partial t^{2}} \\
C_{r}\left(\frac{\partial^{2} u}{\partial r^{2}}+\frac{1}{r} \frac{\partial u}{\partial r}\right)-C_{\theta} \frac{u}{r^{2}}=\rho h \frac{\partial^{2} u}{\partial t^{2}} \\
C_{k}\left(\frac{\partial^{2} v}{\partial r^{2}}+\frac{1}{r} \frac{\partial v}{\partial r}-\frac{v}{r^{2}}\right)=\rho h \frac{\partial^{2} v}{\partial t^{2}} \\
D_{k}\left(\frac{\partial^{2} \psi}{\partial r^{2}}+\frac{1}{r} \frac{\partial \psi}{\partial r}-\frac{\psi}{r^{2}}\right)-K h G_{\theta z} \psi=-\rho \frac{h^{3}}{12} \frac{\partial^{2} \psi}{\partial t^{2}}
\end{gathered}
$$

Here

$$
\begin{aligned}
& D_{r}=\frac{h^{3}}{12} B_{r}, \quad D_{\theta}=\frac{h^{3}}{12} B_{\theta}, \quad D_{k}=\frac{h^{3}}{12} B_{k}, \quad C_{r}=h B_{r}, \quad C_{\theta}=h B_{\theta}, \quad C_{k}=h B_{k}, \\
& D_{r \theta}=D_{r} \sigma_{\theta}+2 D_{k}, \\
& B_{r}=\frac{E_{r}}{1-\sigma_{r} \sigma_{\theta}}, B_{\theta}=\frac{E_{\theta}}{1-\sigma_{r} \sigma_{\theta}}, B_{k}=G_{r \theta}, \\
& E_{r} \sigma_{r}=E_{\theta} \sigma_{\theta}, K=\frac{5}{6},
\end{aligned}
$$

$D_{r}, D_{\theta}$ and $C_{r}, C_{\theta}$ are the stiffness of bending and tension-compression for the $r, \theta$ directions (along and across the rail, respectively); $D_{k}$ - torsional rigidity; $C_{k}-$ shear stiffness; $E_{r}, E_{\theta}$ and $\sigma_{r}, \sigma_{\theta}$ - elastic modulus and Poisson's ratio for $r, \theta$ directions; $G_{r z}, G_{\theta z}$ - shear modulus in the $r z$ and $\theta z$ planes, respectively; $w(r, \theta)$ - the normal displacement of the median plane, $u(r, \theta)$ and $v(r, \theta)$ are the tangential displacements of the median surface along the coordinates $r, \theta$, respectively; $\varphi(r, \theta)$ and $\psi(r, \theta)$ - arbitrary sought functions of the coordinates $r, \theta$.

In order to determine the contact force and dynamic deflection, it is necessary to determine the transverse displacement $w(t)$, which is included in the system of equations (2a) and (2b). The remaining equations are independent subsystems that are solved separately from each other. The functions determined according to relations $(2 \mathrm{c})-(2 \mathrm{e})$ do not affect the considered dynamic characteristics, therefore, only the system of equations (2a) and (2b) will be considered below. The coefficients of the power expansion for the desired function can be obtained by using the methodology, according to which the radial series are substituted in (1), and then the factors of equal degrees of time in the left and 
right sides of the expression are equated.

As a result, a system of recurrent differential equations is obtained from the equations of motion (2a), (2b) for determining the jumps of the sought quantities up to arbitrary constants:

$$
\begin{aligned}
& \left(1-\frac{\rho G^{2}}{B_{r}}\right) \omega_{\varphi(k+1)}=2 \frac{\delta \omega_{\varphi(k)}}{\delta t}+G r^{-1} \omega_{\varphi(k)}+b_{r} G X_{w(k)}+F_{\varphi(k-1)}, \\
& \left(1-\frac{\rho G^{2}}{K G_{r z}}\right) X_{w(k+1)}=2 \frac{\delta X_{w(k)}}{\delta t}+G r^{-1} X_{w(k)}-G \omega_{\varphi(k)}+F_{w(k-1)},
\end{aligned}
$$

the following notation is adopted $X_{w(k)}=\left[w_{,(k+1)}\right], \omega_{\varphi(k)}=\left[\varphi_{,(k+1)}\right]$, $b_{r}=h K G_{r z} D_{r}^{-1}, \quad r=r_{0}+G t$,

$$
\begin{gathered}
F_{\varphi(k-1)}=-\frac{\delta^{2} \omega_{\varphi(k-1)}}{\delta t^{2}}-G r^{-1} \frac{\delta \omega_{\varphi(k-1)}}{\delta t}+G^{2} r^{-2} \frac{E_{\theta}}{E_{r}} \omega_{\varphi(k-1)}-b_{r} G \frac{\delta X_{w(k-1)}}{\delta t}+b_{r} G^{2} \omega_{\varphi(k-1)}, \\
F_{w(k-1)}=-\frac{\delta^{2} X_{w(k-1)}}{\delta t^{2}}-G r^{-1} \frac{\delta X_{w(k-1)}}{\delta t}+G \frac{\delta \omega_{\varphi(k-1)}}{\delta t}+G^{2} r^{-1} \omega_{\varphi(k-1)} .
\end{gathered}
$$

Herein after, let us restrict ourselves to five members of the radial series for the desired functions, which will allow determining them with the necessary accuracy.

Assuming in (3) - (4) $k=-1,0,1,2,3$, the jumps of the corresponding order on the first wave are obtained:

$$
\begin{gathered}
\rho G^{(1) 2}=B_{r}, X_{w(0)}^{(1)}=0 \\
\omega_{\varphi(0)}^{(1)}=c_{0}^{(1)} r_{1}^{-1 / 2}, \quad X_{w(1)}^{(1)}=-\frac{G^{(1)}}{f_{r}} c_{0}^{(1)} r_{1}^{-1 / 2}, \\
\omega_{\varphi(1)}^{(1)}=c_{1}^{(1)} r_{1}^{-1 / 2}+\frac{1}{2}\left(\frac{E_{\theta}}{E_{r}}-\frac{1}{4}\right) G^{(1)} c_{0}^{(1)} r_{1}^{-3 / 2}-\frac{1}{2} G^{(1)} \frac{b_{r}}{e_{r}} c_{0}^{(1)} r_{1}^{1 / 2}, \\
X_{w(2)}^{(1)}=-\frac{G^{(1)}}{f_{r}}\left[c_{1}^{(1)} r_{1}^{-1 / 2}+\frac{1}{2}\left(\frac{E_{\theta}}{E_{r}}-\frac{5}{4}\right) G^{(1)} c_{0}^{(1)} r_{1}^{-3 / 2}-\frac{1}{2} G^{(1)} \frac{b_{r}}{e_{r}} c_{0}^{(1)} r_{1}^{1 / 2}\right], \\
\omega_{\varphi(2)}^{(1)}=c_{2}^{(1)} r_{1}^{-1 / 2}+\frac{1}{2}\left(\frac{E_{\theta}}{E_{r}}-\frac{1}{4}\right) G^{(1)} c_{1}^{(1)} r_{1}^{-3 / 2}-\frac{1}{2} G^{(1)} \frac{b_{r}}{e_{r}} c_{1}^{(1)} r_{1}^{1 / 2}+ \\
G^{(1) 2} \frac{b_{r}^{2}}{e_{r}^{2}} c_{0}^{(1)} r_{1}^{3 / 2}-\frac{1}{8}\left(\frac{9}{4}-\frac{E_{\theta}}{E_{r}}\right)\left(\frac{E_{\theta}}{E_{r}}-\frac{1}{4}\right) G^{(1) 2} c_{0}^{(1)} r_{1}^{-5 / 2}, \\
X_{w(3)}^{(1)}=-\frac{G^{(1)}}{f_{r}}\left[c_{2}^{(1)} r_{1}^{-1 / 2}+\frac{1}{2}\left(\frac{E_{\theta}}{E_{r}}-\frac{5}{4}\right) G^{(1)} c_{1}^{(1)} r_{1}^{-3 / 2}-\frac{1}{2} G^{(1)} \frac{b_{r}}{e_{r}} c_{1}^{(1)} r_{1}^{1 / 2}-\right. \\
-\left(\frac{E_{\theta}}{E_{r}}-1\right) G^{(1) 2} \frac{1}{f_{r}} c_{0}^{(1)} r_{1}^{-5 / 2}+\frac{1}{8}\left(\frac{E_{\theta}}{E_{r}}-\frac{1}{4}\right)^{2} G^{(1) 2} c_{0}^{(1)} r_{1}^{-5 / 2}- \\
\left.-\left(\frac{1}{f_{r}}-\frac{3}{4}\right) G^{(1) 2} \frac{b_{r}}{e_{r}} c_{0}^{(1)} r_{1}^{-1 / 2}+\frac{1}{8} G^{(1) 2} \frac{b_{r}^{2}}{e_{r}^{2}} c_{0}^{(1)} r_{1}^{3 / 2}\right]
\end{gathered}
$$


here $f_{r}=1-\frac{B_{r}}{K G_{r z}}=1-\frac{G^{(1) 2}}{G^{(2) 2}}<0 \frac{1}{e_{r}}=1-\frac{1}{f_{r}}, e_{r}=1-\frac{K G_{r z}}{B_{r}}=1-\frac{G^{(2) 2}}{G^{(1) 2}}>0$.

Similarly, the following is obtained according to the second wave

$$
\begin{gathered}
\rho G^{(2) 2}=K G_{r z}, \omega_{\varphi(0)}^{(2)}=0, \\
X_{w(0)}^{(2)}=c_{0}^{(2)} r_{2}^{-1 / 2}, \quad \omega_{\varphi(1)}^{(2)}=G^{(2)} \frac{b_{r}}{e_{r}} c_{0}^{(2)} r_{2}^{-1 / 2}, \\
X_{w(1)}^{(2)}=c_{1}^{(2)} r_{2}^{-1 / 2}-\frac{1}{8} G^{(2)} c_{0}^{(2)} r_{2}^{-3 / 2}+\frac{1}{2} G^{(2)} \frac{b_{r}}{e_{r}} c_{0}^{(2)} r_{2}^{1 / 2},
\end{gathered}
$$




$$
\begin{aligned}
& \omega_{\varphi(2)}^{(2)}=G^{(2)} \frac{b_{r}}{e_{r}}\left[c_{1}^{(2)} r_{2}^{-1 / 2}+\frac{3}{8} G^{(2)} c_{0}^{(2)} r_{2}^{-3 / 2}+\frac{1}{2} G^{(2)} \frac{b_{r}}{e_{r}} c_{0}^{(2)} r_{2}^{1 / 2}\right], \\
& X_{w(2)}^{(2)}=c_{2}^{(2)} r_{2}^{-1 / 2}-\frac{1}{8} G^{(2)} c_{1}^{(2)} r_{2}^{-3 / 2}+\frac{1}{2} \frac{b_{r}}{e_{r}} G^{(2)} c_{1}^{(2)} r_{2}^{1 / 2}+ \\
& +\frac{1}{8} \frac{b_{r}^{2}}{e_{r}^{2}} G^{(2) 2} c_{0}^{(2)} r_{2}^{3 / 2}+\frac{9}{128} G^{(2) 2} c_{0}^{(2)} r_{2}^{-5 / 2}, \\
& \omega_{\varphi(3)}^{(2)}=G^{(2)} \frac{b_{r}}{e_{r}}\left\{c_{2}^{(2)} r_{2}^{-1 / 2}+\frac{3}{8} G^{(2)} c_{1}^{(2)} r_{2}^{-3 / 2}+\frac{1}{2} G^{(3)} \frac{b_{r}}{e_{r}} c_{1}^{(2)} r_{2}^{1 / 2}+\right. \\
& +\frac{1}{8} \frac{b_{r}^{2}}{e_{r}^{2}} G^{(2) 2} c_{0}^{(2)} r_{2}^{3 / 2}-\left[\frac{1}{e_{r}}\left(1-\frac{E_{\theta}}{E_{r}}\right)+\frac{15}{128}\right] G^{(2) 2} c_{0}^{(2)} r_{2}^{-5 / 2}+ \\
& \left.+\left(\frac{1}{e_{r}}+\frac{3}{4}\right) \frac{b_{r}}{e_{r}} G^{(2) 2} c_{0}^{(2)} r_{2}^{-1 / 2}\right\}, \\
& X_{w(3)}^{(2)}=c_{3}^{(2)} r_{2}^{-1 / 2}-\frac{1}{8} G^{(2)} c_{2}^{(2)} r_{2}^{-3 / 2}+\frac{1}{2} \frac{b_{r}}{e_{r}} G^{(2)} c_{2}^{(2)} r_{2}^{1 / 2}+\frac{1}{8} \frac{b_{r}^{2}}{e_{r}^{2}} G^{(2) 2} c_{1}^{(2)} r_{2}^{3 / 2}+ \\
& +\frac{9}{128} G^{(2) 2} c_{1}^{(2)} r_{2}^{-5 / 2}+\frac{1}{2} \frac{b_{r}}{e_{r}}\left[\frac{1}{e_{r}}\left(1-\frac{E_{\theta}}{E_{r}}\right)-\frac{9}{128}\right] G^{(2) 3} c_{0}^{(2)} r_{2}^{-3 / 2}+ \\
& +\frac{1}{2} \frac{b_{r}^{2}}{e_{r}^{2}}\left(\frac{1}{e_{r}}+\frac{9}{32}\right) G^{(2) 3} c_{0}^{(2)} r_{2}^{1 / 2}+\frac{1}{48} \frac{b_{r}^{3}}{e_{r}^{3}} G^{(2) 3} c_{0}^{(2)} r_{2}^{5 / 2}-\frac{75}{1024} G^{(2) 3} c_{0}^{(2)} r_{2}^{-7 / 2} \text {, } \\
& \omega_{\varphi(4)}^{(2)}=G^{(2)} \frac{b_{r}}{e_{r}}\left\{c_{3}^{(2)} r_{2}^{-1 / 2}+\frac{3}{8} G^{(3)} c_{2}^{(2)} r_{2}^{-3 / 2}+\frac{1}{2} G^{(2)} \frac{b_{r}}{e_{r}} c_{2}^{(2)} r_{2}^{1 / 2}+\right. \\
& +\frac{1}{8} \frac{b_{r}^{2}}{e_{r}^{2}} G^{(2) 2} c_{1}^{(2)} r_{2}^{3 / 2}-\left[\frac{1}{e_{r}}\left(1-\frac{E_{\theta}}{E_{r}}\right)+\frac{15}{128}\right] G^{(2) 2} c_{1}^{(2)} r_{2}^{-5 / 2}+ \\
& +\left(\frac{1}{e_{r}}+\frac{3}{4}\right) \frac{b_{r}}{e_{r}} G^{(2) 2} c_{1}^{(2)} r_{2}^{-1 / 2}+\left(1-\frac{E_{\theta}}{E_{r}}\right)\left(\frac{3}{8}-\frac{4}{e_{r}}\right) \frac{1}{e_{r}} G^{(2) 3} c_{0}^{(2)} r_{2}^{-7 / 2}+ \\
& +\frac{105}{1024} G^{(2) 3} c_{0}^{(2)} r_{2}^{-7 / 2}+\frac{3}{8}\left(\frac{1}{e_{r}}+\frac{29}{32}\right) \frac{b_{r}}{e_{r}} G^{(2) 3} c_{0}^{(2)} r_{2}^{-3 / 2}+ \\
& \left.+\left(\frac{1}{e_{r}}+\frac{29}{64}\right) \frac{b_{r}^{2}}{e_{r}^{2}} G^{(2) 3} c_{0}^{(2)} r_{2}^{1 / 2}+\frac{b_{r}^{3}}{48 e_{r}^{3}} G^{(2) 3} c_{0}^{(2)} r_{2}^{5 / 2}\right\}, \\
& X_{w(4)}^{(2)}=c_{4}^{(2)} r_{2}^{-1 / 2}-\frac{1}{8} G^{(2)} c_{3}^{(2)} r_{2}^{-3 / 2}+\frac{1}{2} \frac{b_{r}}{e_{r}} G^{(2)} c_{3}^{(2)} r_{2}^{1 / 2}+\frac{1}{8} \frac{b_{r}^{2}}{e_{r}^{2}} G^{(2) 2} c_{2}^{(2)} r_{2}^{3 / 2}+ \\
& +\frac{9}{128} G^{(2) 2} c_{2}^{(2)} r_{2}^{-5 / 2}+\frac{1}{2} \frac{b_{r}}{e_{r}}\left[\frac{1}{e_{r}}\left(1-\frac{E_{\theta}}{E_{r}}\right)-\frac{9}{128}\right] G^{(2) 3} c_{1}^{(2)} r_{2}^{-3 / 2}+ \\
& +\frac{1}{2} \frac{b_{r}^{2}}{e_{r}^{2}}\left(\frac{1}{e_{r}}+\frac{9}{32}\right) G^{(2) 3} c_{1}^{(2)} r_{2}^{1 / 2}+\frac{1}{48} \frac{b_{r}^{3}}{e_{r}^{3}} G^{(2) 3} c_{1}^{(2)} r_{2}^{5 / 2}-\frac{75}{1024} G^{(2) 3} c_{1}^{(2)} r_{2}^{-7 / 2}-
\end{aligned}
$$




$$
\begin{gathered}
-\frac{1}{4}\left[\left(1-\frac{E_{\theta}}{E_{r}}\right)\left(\frac{4}{e_{r}}-\frac{3}{4}\right) \frac{1}{e_{r}}-\frac{117}{1024}\right] \frac{b_{r}}{e_{r}} G^{(2) 4} c_{0}^{(2)} r_{2}^{-5 / 2}+ \\
+\frac{1}{4}\left(\frac{1}{e_{r}}+\frac{25}{24}\right) \frac{b_{r}^{3}}{e_{r}^{3}} G^{(2) 4} c_{0}^{(2)} r_{2}^{3 / 2}+\frac{1}{384} \frac{b_{r}^{4}}{e_{r}^{4}} G^{(2) 4} c_{0}^{(2)} r_{2}^{7 / 2}+\frac{3675}{16384} G^{(2) 4} c_{0}^{(2)} r_{2}^{-9 / 2} .
\end{gathered}
$$

The jumps found allow writing down the expressions for the desired functions $W$ (dynamic deflection speed of the track foundation slab) and $Q_{r}$ (cutting force at the boundary of the contact region) in the form of line segments (22), (23) when replacing the module $\mu$ by $G_{r z}$ and the jumps $\omega_{(i)}^{(\alpha)}, X_{(i)}^{(\alpha)}$ by $\omega_{\varphi(i)}^{(\alpha)}, X_{w(i)}^{(\alpha)} \quad(\alpha=1,2)(i=0,1, \ldots 4)$, respectively. Thus, the desired functions were determined up to arbitrary constants $c_{i}^{(\alpha)}(\alpha$ $=1,2)(i=0,1, \ldots 4)$, which are obtained according to the boundary conditions.

$$
\begin{gathered}
W \cong \sum_{\alpha=1}^{2} \sum_{k=0}^{4} \frac{1}{k !} X_{(k)}^{(\alpha)}\left(y_{\alpha}\right)^{k} H\left(y_{\alpha}\right) \\
Q_{r} \cong K \mu h \sum_{\alpha=1}^{2} \sum_{k=0}^{4} \frac{1}{k !}\left(-X_{(k)}^{(\alpha)} G^{(\alpha)-1}+\frac{\delta X_{(k-l)}^{(\alpha)}}{\delta t} G^{(\alpha)-1}-\omega_{(k-1)}^{(\alpha)}\right)\left(y_{\alpha}\right)^{k} H\left(y_{\alpha}\right)
\end{gathered}
$$

here $y_{\alpha}=t-\left(r-r_{0}\right) G^{(\alpha)-1}$, values $X_{(k)}^{(\alpha)}, \omega_{(k)}^{(\alpha)}$ and their $\delta$-derivatives are calculated for $y_{\alpha}=0$.

Arbitrary derivatives $c_{i}^{(\alpha)}(\alpha=1,2)(i=0,1, \ldots 4)$ can be determined according to the equation system (1), after substituting the segments of the radial series (22), (23), a power time expansion for buckling $\alpha$, expressions for the interaction force between the wheel and the rail, and subsequent equalization of the resulting factors at the same degrees of $t$.

For numerical studies, the resulting correlations between the force of the dynamic interaction between the wheel and the rail and the vertical track foundation slab movement is presented in a following dimensionless form:

$$
\begin{gathered}
\tilde{w}(\tilde{t})=\frac{\tilde{E} \tilde{V}}{\pi \tilde{h}}\left[\frac{\tilde{t}^{3}}{6}-\left(1+\frac{G^{(2)}}{G^{(1)}}\right) \frac{\tilde{t}^{4}}{12}+\left[3\left(1+\frac{G^{(2)}}{G^{(1)}}\right)^{2}-\frac{\tilde{E}}{\pi \tilde{h}}(\tilde{m}+2)\right] \frac{\tilde{t}^{5}}{120}+\right. \\
+\left[\frac{2 \tilde{E}}{\pi \tilde{h}}\left(1+\frac{G^{(2)}}{G^{(1)}}\right)(\tilde{m}+4)-4\left(1+\frac{G^{(2)}}{G^{(1)}}\right)^{3}+\right. \\
\left.\left.+\frac{1}{4}\left(1+\frac{G^{(2) 3}}{G^{(1) 3}}\right)+\frac{12 G^{(2) 2}}{\tilde{h}^{2} G^{(1) 2}}\left(1-\frac{G^{(2) 3}}{G^{(1) 3}}\right)\left(1-\frac{G^{(2) 2}}{G^{(1) 2}}\right)^{-1}-\left(\frac{E_{\theta}}{E_{r}}-1\right) \frac{G^{(2)}}{G^{(1)}}\left(1-\frac{G^{(2)}}{G^{(1)}}\right)^{-1}\right] \frac{\tilde{t}^{6}}{720}\right], \\
\tilde{P}(\tilde{t})=\tilde{V}\left\{\tilde{t}-\frac{\tilde{E}}{\pi \tilde{h}}(\tilde{m}+2) \frac{\tilde{t}^{3}}{6}+\frac{\tilde{E}}{\pi \tilde{h}}\left(1+\frac{G^{(2)}}{G^{(1)}}\right) \frac{\tilde{t}^{4}}{6}-\right.
\end{gathered}
$$



here

$$
\begin{gathered}
-\frac{\tilde{E} \tilde{h}}{\pi \tilde{h}}\left[\left(1+\frac{G^{(2)}}{G^{(1)}}\right)^{2}-\frac{\tilde{E}}{6 \pi \tilde{h}}(\tilde{m}+2)^{2}\right] \frac{\tilde{t}^{5}}{20}+ \\
+\frac{\tilde{E}}{\pi \tilde{h}}\left[-\frac{\tilde{E}}{3 \pi \tilde{h}}\left(1+\frac{G^{(2)}}{G^{(1)}}\right)(\tilde{m}+2)+\frac{1}{3}\left(1+\frac{G^{(2)}}{G^{(1)}}\right)^{3}-\frac{1}{48}\left(1+\frac{G^{(2) 3}}{G^{(1) 3}}\right)-\right. \\
\left.\left.-\frac{G^{(2) 2}}{\tilde{h}^{2} G^{(1) 2}}\left(1-\frac{G^{(2) 3}}{G^{(1) 3}}\right)\left(1-\frac{G^{(2) 2}}{G^{(1) 2}}\right)^{-1}+\frac{1}{12}\left(\frac{E_{\theta}}{E_{r}}-1\right) \frac{G^{(2)}}{G^{(1)}}\left(1-\frac{G^{(2)}}{G^{(1)}}\right)^{-1}\right] \frac{\tilde{t}^{6}}{30}\right], \\
\text { here } \tilde{w}(\tilde{t})=\frac{w(t)}{r_{0}}, \frac{G^{(2)}}{G^{(1)}}=\sqrt{\frac{K G_{r z}}{B_{r}}}=\sqrt{\frac{K G_{r z}\left(1-\sigma_{r} \sigma_{\theta}\right)}{E_{r}}}, \tilde{E}=\frac{E_{1} / r_{0}}{B_{r}} .
\end{gathered}
$$

\section{Results and discussion}

These correlations allow evaluating the effect of the orthotropic properties of the reinforced concrete foundation slab of a ballastless track, the speed of rolling stock, defects in wheel sets or rail bars (increases the vertical contact force between the wheel and rail). It is also possible to evaluate the technical content parameters of the track section, for example, the rigidity of the base during lining and tamping works [29, 30, 31].

Figure 4 shows the graphical dependences of the maximum contact force $\tilde{P}$ on the vertical component of the dynamic contact speed of the wheel and rail $\tilde{V}$ (approximately corresponds to the rolling stock speed from 0 to $200 \mathrm{~km} / \mathrm{h}$ ). The ballast-free track design has the following parameters: axle load -20 tons, reinforced concrete slab thickness -20 $\mathrm{cm}$. The contact stiffness of the rail and half ties was taken according to the ARS-4 baseplate, the remaining characteristics were adopted as standard for the Russian railways. Curve 1 in Fig. 4 is plotted for the given dimensionless characteristic of the railway track stiffness $\tilde{E}=90 \cdot 10^{-6}$, curve $2-\tilde{E}=40 \cdot 10^{-6}$, curve $3-\tilde{E}=10 \cdot 10^{-6}$, curve $4-\tilde{E}=$ $1 \cdot 10^{-6}$, curve $5-\tilde{E}=0.05 \cdot 10^{-6}$, curve $6-\tilde{E}=0.02 \cdot 10^{-6}$. The presented values of the given dimensionless characteristic of the railway track stiffness approximately correspond to changes in the upper track structure stiffness when using track structures both of ballastless type (curves 1,2) and traditional type structures on a ballast prism with varying degrees of rubble embedding and compacting (curves $3,4,5,6$ ).

It can be noted that with an $\tilde{E}$ increase, the maximum forces of interaction between the wheel and the rail increase. With $\tilde{E}$ decrease, interaction forces decrease in comparison with a certain straight-line dependence corresponding to a railway track on a ballast maintained in a normative state [32]. With an increase in the absolute $\tilde{E}$ value, the correlation between the interaction force on the vertical component of the rolling stock speed $\tilde{V}$ is increasingly affected by the nonlinear properties of the railway structure. 


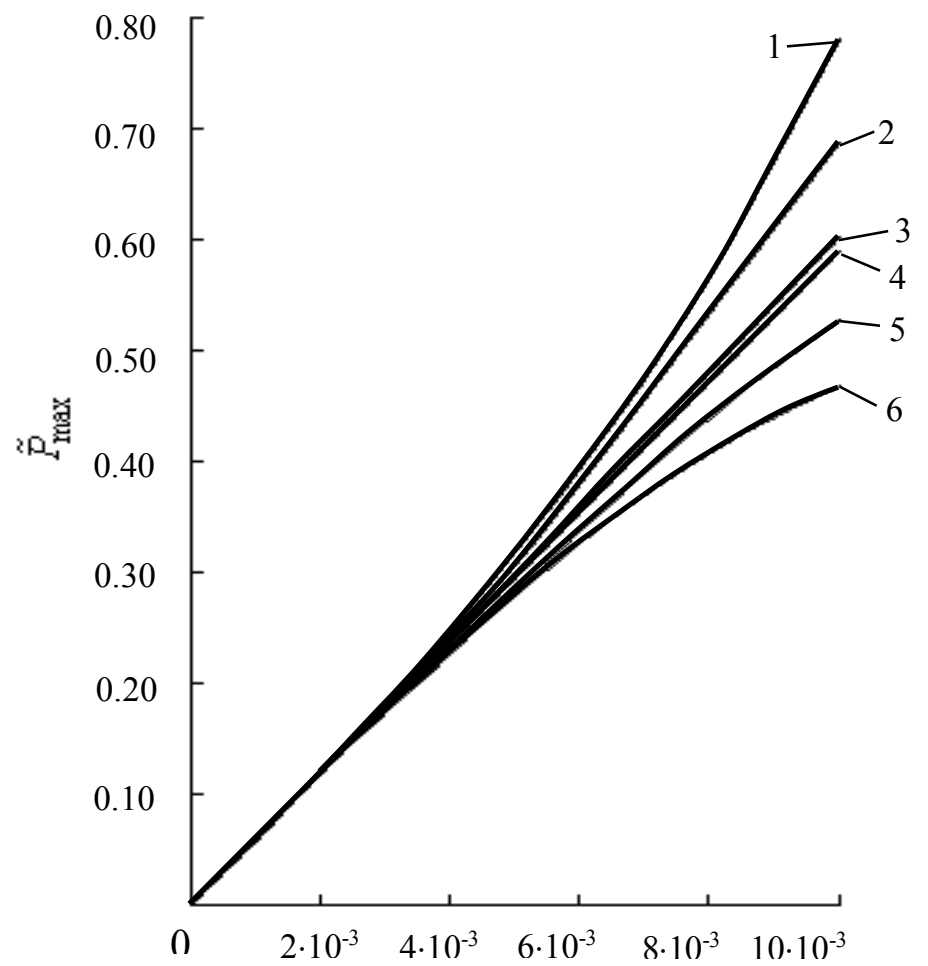

Fig. 4. The dependence of the dimensionless maximum interaction force between the wheel and the rail on the vertical speed component of the dynamic wheel and rail contact $\tilde{V}$ for various values of the reduced dimensionless characteristic of the railway track stiffness $\tilde{E}$

\section{Conclusions}

After analyzing the obtained analytical and graphical correlations between the dynamic and kinematic parameters of the railway track construction behavior, a number of following qualitative conclusions can be made:

1) The value of the maximum interaction force between the wheel and the rail significantly decreases with decreasing track stiffness at the point of contact with the wheel pair.

2) The maximum interaction force between the wheel and the rail increases with the increase in size of the contact area, which is quite significant in the presence of defects in wheelsets, such as slid flats and scaled wheels, thickness of the foundation slab and the speed of the rolling stocks.

3) Non-linear rigidity parameters of the track structure begin to significantly impact the interaction force of the wheel and rail in the middle of the dynamic contact process, as well as with an increase in rolling stock speeds.

4) The stiffness parameters of the railway track can both increase the maximum interaction force between the wheel and the rail, making its graphic dependence rigid (the second derivative is positive), and also reduce it with a soft characteristic of non-linearity (the second derivative is negative) compared to the value for the normative state of the railway ballast construction in the present study. 


\section{References}

1. V. Korolev, A. Loktev et al. IOP Conference Series: Earth and Environmental Science, 403, DOI: 10.1088/1755-1315/403/1/012194

2. A. Loktev, V. Korolev et al. IOP Conf. Series: Materials Science and Engineering, 463, 032019 (2018) DOI: 10.1088/1757-899X/463/3/032019

3. S. Abrate, Applied Mechanics Reviews. 44 (4), 155-190 (1991).

4. S. Abrate, Impact on composite structures (Cambridge University Press, 1998)

5. J.D., Achenbach D.P. Reddy Z. Angew. Math. Phys. (18). 141-144 (1967).

6. A. Savin, V. Korolev et al. TransSiberia 2019: VIII International Scientific Siberian Transport Forum, 797-808 DOI: 10.1007/978-3-030-37916-2_78

7. A.V. Savin, V.V. Korolev, I.V. Shishkina. IOP Conf. Series: Materials Science and Engineering, 687, 022035 (2019) DOI: 10.1088/1757-899X/687/2/022035

8. A. Savin, A. Kogan et al. International Journal of Innovative Technology and Exploring Engineering, 8(7), 2325-2328 (2019)

9. M.M. Al-Mousawi, Applied Mechanics Reviews. 39(6), 853-864 (1986).

10. M.E. Backman, W. Goldsmith, International Journal of Engineering Sciences. 16, 1-99 (1978).

11. V. Korolev. TransSiberia 2019: VIII International Scientific Siberian Transport Forum, 175-187 DOI: 10.1007/978-3-030-37919-3_17

12. A. Loktev, V. Korolev et al. Vestnik of the Railway Research Institute, 77(6), 331-336 (2018) DOI: 10.21780/2223-9731-2018-77-6-331-336

13. V.A. Bazhenov, O.S. Pogorelova et al., Strength of Materials. 40(6).656 - 662 (2008).

14. W.J. Cantwell, J. Morton Composites. 22(5). 347-362 (1991).

15. A. Loktev, V. Korolev et al. Procedia Engineering, 189, 133-137 (2017) DOI: 10.1016/j.proeng.2017.05.022

16. A. Loktev, V. Korolev et al. Advances in Intelligent Systems and Computing, 982, 325-336 (2020) DOI: 10.1007/978-3-030-19756-8_30

17. S. Chattopadhyay Journal of the Acoustical Society of America. 82(2), 493-497 (1987).

18. A.A. Loktev, E.A. Gridasova, E.V. Zapol'nova Contemporary Engineering Sciences. 8(17-20), 799-807 (2015).

19. V. Goremikins, D. Serdjuks, K. Buka-Vaivade, L. Pakrastins, N. Vatin. Prediction of behaviour of prestressed suspension bridge with timber deck panels. Baltic Journal of Road and Bridge Engineering. 2017. 12(4). Pp. 234-240. DOI:10.3846/bjrbe.2017.29

20. B. Glusberg, A. Savin et al. Advances in Intelligent Systems and Computing, 982, 556570 (2020) DOI: 10.1007/978-3-030-19756-8_53

21. B. Glusberg, A. Savin et al. Advances in Intelligent Systems and Computing, 982, 571587 (2020) DOI: 10.1007/978-3-030-19756-8_54

22. V. Korolev. TransSiberia 2019: VIII International Scientific Siberian Transport Forum, 621-638 DOI: 10.1007/978-3-030-37916-2_60

23. A.A. Loktev, E.A. Gridasova, A.V. Sycheva, R.N. Stepanov, Contemporary Engineering Sciences, 8(21-24), 955-962 (2015)

24. A.P. Christoforou, A.A. Elsharkawy, L.H. Guedouar Computers and Structures. 79, 2607-2619 (2001). 
25. A. Loktev, V. Korolev et al. IOP Conf. Series: Materials Science and Engineering, 463, 032018 (2018) DOI: 10.1088/1757-899X/463/3/032018

26. A. Loktev, V. Korolev et al. Vestnik of the Railway Research Institute, 77(2), 77-83 (2018) DOI: 10.21780/2223-9731-2018-77-2-77-83

27. A. Lyudagovsky, A. Loktev, V. Korolev et al. E3S Web of Conferences, 110, 01017 (2019) DOI: 10.1051/e3sconf/201911001017

28. B. Glusberg, A. Loktev et al. Advances in Intelligent Systems and Computing, 982, 337-345 (2020) DOI: 10.1007/978-3-030-19756-8_31

29. B. Glusberg, V. Korolev et al. MATEC Web of Conferences, 239, 01054 (2018) DOI: $10.1051 /$ matecconf $/ 201823901054$

30. A. Savin, O. Suslov et al. TransSiberia 2019: VIII International Scientific Siberian Transport Forum, 648-654 DOI: 10.1007/978-3-030-37916-2_62

31. B. Glusberg, V. Korolev et al. E3S Web of Conferences, 138, 01017 (2019) DOI: $10.1051 / \mathrm{e} 3$ sconf $/ 201913801017$

32. I. Shishkina. TransSiberia 2019: VIII International Scientific Siberian Transport Forum, 834-844, DOI: 10.1007/978-3-030-37916-2_82 